\title{
Cuando el espacio conceptualizado se encuentra con el espacio vivido. Los proyectos territoriales de desarrollo como complejos procesos de «traducción»
}

\author{
Brice de Reymaeker \\ Universitat Autònoma de Barcelona. Departament de Geografia \\ dereymaekerbrice@gmail.com
}

Recepción: enero de 2011

Aceptación: mayo de 2011

\section{Resumen}

En el presente artículo, se muestran las líneas teóricas y las sucesivas propuestas metodológicas que informan mi tentativa de estudiar, bajo un enfoque geográfico inspirado en el constructivismo social, los proyectos de cooperación para el desarrollo territorial. Después de presentar dichos proyectos como conjuntos dinámicos y en relación entre sí de concepciones, prácticas y representaciones espaciales, se revisan diferentes aportaciones esenciales, tanto de la geografía postcolonial y feminista, como de la perspectiva no representacional. En efecto, dichas aportaciones pueden servir de punto de partida para conceptualizar, de manera más sutil, el proceso de traducción o de negociación que tiene lugar no sólo entre los actores involucrados en los proyectos de cooperación para el desarrollo territorial, sino también entre sus diferentes espacios concebidos, percibidos y vividos.

Palabras clave: cooperación para el desarrollo territorial; proceso de traducción; dispositivo de saber-poder; Lefebvre.

Resum. Quan l'espai conceptualitzat es troba amb l'espai viscut. Els projectes territorials de desenvolupament com a complexos processos de "traducció"

En el present article, s'hi mostren les línies teòriques i les propostes metodològiques successives que informen la meva temptativa d'estudiar, sota un enfocament geogràfic inspirat en el constructivisme social, els projectes de cooperació per al desenvolupament territorial. Després de presentar aquests projectes com a conjunts dinàmics i en relació entre si de concepcions, pràctiques i representacions espacials, s'hi revisen diferents aportacions essencials, tant de la geografia postcolonial i feminista, com de la perspectiva no representacional. En efecte, aquestes aportacions poden servir de punt de partida per conceptualitzar, de manera més subtil, el procés de traducció o de negociació que té lloc no només entre els actors involucrats en els projectes de cooperació per al desenvolupament territorial, sinó també entre els diversos espais concebuts, percebuts i viscuts.

Paraules clau: cooperació per al desenvolupament territorial; procés de traducció; dispositiu de saber-poder; Lefebvre. 
Résumé. Quand l'espace conçu rencontre l'espace vécu. Les projets de développement territorial en tant que processus complexes de "traduction»

Dans cet article sont présentées les lignes théoriques et les successives propositions méthodologiques qui guident ma tentative d'étudier, à travers une approche géographique inspirée du constructivisme social, les projets de coopération au développement territorial. Après la présentation de ces projets comme des ensembles dynamiques et en relation entre eux de conceptions, pratiques et représentations spatiales, différentes apports essentielles tant de la géographie postcoloniale et féministe, comme de la perspective non-représentationnelle sont revisités. De fait, ces apports peuvent servir de point de départ pour conceptualiser, de manière plus subtile, le processus de traduction ou de négociation qui prend place non seulement entre les différents acteurs impliqués dans les projets de développement territorial, sinon également entre ses différents espaces conçus, perçus et vécus.

Mots clé: coopération au développement territorial; processus de traduction; dispositif de savoir-pouvoir; Lefebvre.

Abstract. When the designed space meets the lived space. The territorial development projects as complex processes of "translation»

In the present article the theoretical lines and the successive methodological proposals that guide my attempt to study the projects of cooperation for territorial development, under a geographic approach inspired by social constructivism, are presented. After presenting those projects like dynamic wholes in relation between themselves of spatial conceptions, practices and representations, different essential contributions of postcolonial and feminist geography as well as of the no representational perspective are reviewed. In fact those contributions may serve as departure point for the conceptualization, in a more subtle way, of the process of translation or negotiation that occurs not only between the different actors engaged in the territorial development projects but also between their different conceived, perceived and lived spaces.

Key words: cooperation for territorial development; process of translation; apparatus of knowledge-power; Lefebvre.

\begin{aligned} & \multicolumn{2}{c}{ Sumario } \\ & Introducción $\begin{array}{l}\text { Feminismo, postcolonialismo y teoría no } \\ \text { representacional: de la deconstrucción }\end{array} \\ & \begin{aligned} \text { Los proyectos de desarrollo territorial } \\ \text { como encuentros de dispositivos de } \\ \text { desarrollo territorial: el principio }\end{aligned} \begin{array}{l}\text { Conclusiones } \\ \text { de traducción y el tríptico espacial }\end{array} \\ &$ Referencias bibliográficas \end{aligned}

\section{Introducción}

Il ne peut exister d'identité essentielle, fût-ce par le «territoire» qui n'est qu'une circonstance d'association plus ou moins voulue et maîtrisée. Il faudra faire droit à ce qui relève du politique, du culturel et du social (sociétal) dans la constitution d'entités naturalisées par référence à la terre. (Retaillé, 2007: 168) 
En aras de aprehender lo que se entiende comúnmente en el mundo de la cooperación como proyectos de desarrollo, resulta útil acercarse al enfoque del marco lógico (EML en lo que sigue), ya que dicho enfoque ha sido adoptado por la mayoría de los actores de la cooperación para el desarrollo - los principales países donantes, las principales organizaciones de cooperación multilateral y regional y, consecuentemente, por la mayoría de los actores promotores de proyectos que son las ONG. El EML, nacido de una necesidad de controlar y evaluar los proyectos de desarrollo en los años sesenta, se presenta en la actualidad como "un método de gestionar un proyecto» a lo largo de todas sus fases y como «un sistema de planificación por objetivos» (Gómez y Sáinz, 2003: 118). Este método se basa en la aplicación de una lógica causal a la diagnosis de problemas y, posteriormente, a la elaboración de una cadena de medios y fines correspondiente. Dicho enfoque se encuentra íntimamente relacionado con la lógica aristotélica con su causalidad lineal y unidireccional, con la descomposición del todo en sus partes, y apuesta claramente por el método inductivo (Calderón, 2008: 11-12). De hecho, en el EML se habla de «árboles de problemas» y de "árboles de objetivos», metáforas que bien reflejan esta idea de linearidad dirigida — de las raíces a la cima, así como la no-interrelación de las diferentes ramas ${ }^{1}$. En cuanto a los efectos prácticos de este nuevo espiritu "managerial», Mawdsley et al. (2002) insisten sobre la uniformización y estandarización de las prácticas de cooperación para el desarrollo proveniente de la profesionalización del sector, tendencia en la cual el EML ha incidido fuertemente.

Mi apuesta en el presente artículo es la de mostrar los proyectos de cooperación territorial, no tanto como respuestas lógicas (enmarcadas en un cuadro conceptual preestablecido y externo a la situación a la cual se dirigen los proyectos) a ciertos problemas, sino como verdaderos ejercicios de creación en común. Para llegar a esta reconceptualización, me apoyo, en el primer apartado, en el principio de traducción que se produce en toda situación social donde "viaja» un objeto o una idea entre diferentes actores (Latour, 1996 y 2005; Said, 1983). Sucesivamente, ya que me interesan específicamente los proyectos de desarrollo territorial, se discute el tríptico espacial de Lefebvre (1974) con un interés particular para las relaciones entre las tres partes y entre los diferentes actores participando en la producción del espacio. En el segundo apartado, discuto las posibles aportaciones de las geografías feminista y postcolonial, así como de la teoría no representacional para el objetivo asignado en el primero. Finalmente, justifico mi propuesta teórica a través de la reivindicación de una obertura radical al «Otro», al devenir y al papel de las prácticas frente a lo discursivo.

1. Es interesante poner de relieve que es justamente en contra del modelo de arborescencia que Deleuze y Guattari introducen el concepto de rizoma que pone en cortocircuito las relaciones jerárquicas entre los varios elementos de un conjunto (Deleuze y Guattari, 1972; Jones, 2008). 


\section{Los proyectos de desarrollo territorial como encuentros de dispositivos de desarrollo territorial: el principio de traducción y el tríptico espacial}

Para Bruno Latour, uno de los exponentes más destacados de la Actor-NetworkTheory o, más bien dicho, de la «sociología de la traducción» (Latour, 2005: 106), lo social no es un dominio aparte, ni tan solo el resultado de «fuerzas sociales», sino un proceso. Lo social entendido como red de elementos dispares - actores humanos y actores no humanos- es siempre un ensamblaje y los elementos que lo forman asumen el papel de intermediarios o el de mediadores ${ }^{2}$ de lo que circula y se produce entre ellos: la progresiva creación de sentido o una cierta fuerza (Latour, 2005: 39). Una de las conclusiones de este enfoque resulta ser para Latour que «la tarea de definir y ordenar lo social debería ser dejada a los mismos actores, y no emprendido por el analista» (Latour, 2005: 23). Este llamamiento se sitúa en la continuación de los estudios etnometodológicos con su insistencia en desconfiar del tratamiento «ad hoc» (es decir, a medida del enfoque teórico y de la tradición pero no a medida del objeto singular estudiado) de los métodos analíticos y explicativos dominantes en su época. También comparte el objetivo de Garfinkel de centrarse en el proceso de construcción del sentido común (Garfinkel, 1967). Otra conclusión importante es que no hay elementos que, viajando por una red, no se traduzcan o se transformen (Latour, $1996 \mathrm{y}$ 2005; Massey, 2003). Me parece importante insistir sintéticamente sobre estos dos elementos cruciales: $a$ ) cuando hablamos de fenómenos sociales, no hay elementos intangibles que puedan imponerse sobre el proceso concreto estudiado, siempre se producen transformaciones; $b$ ) las lógicas de los actores son las que más tienen que ver con el resultado del trabajo efectuado a través de una red.

Edward Said también se ocupa del concepto de traducción, pero lo hace refiriéndose al contexto particular de las relaciones interculturales. Inspirándose en el concepto de "comunidades interpretativas» de Fish (1980), propone, para el estudio del trasplante de ideas, un marco analítico en cuatro etapas: a) el estudio del "punto de origen" y la "entrada en el discurso» de una idea; b) la "distancia recorrida», o sea, "la travesía bajo la presión de diferentes contextos»; c) el conjunto de condiciones de aceptación, resistencia, introducción o tolerancia, y d) la manera en que esta nueva idea acomodada y/o incorporada «viene transformada por sus nuevos usos y su nueva posición en un nuevo tiempo y espacio» (Said, 1983: 226-227). Insistiendo en el mismo proceso de traducción o transformación que Latour, pero aplicándolo a colectivos humanos y no a actores humanos y no humanos, Said se refiere a un contexto similar a lo que encontramos en los proyectos de cooperación al desarrollo - la transculturalidad y postcolonialidad - y nos permite realizar un primer acercamiento metodológico a través de este marco en cuatro fases o puntos de referencia.

2. «Un intermediario [...] es lo que transporta sentido o fuerza sin transformación: definiendo sus inputs es suficiente para definir sus outputs [...] por otra parte [...] el input [de un mediador] nunca es un buen predicador de su output; sus especificidades deben ser presas en consideraciones cada vez. Los mediadores transforman, traducen, distorsionan, y modifican el sentido o los elementos que supuestamente transportan» (Latour, 2005: 39). 
Además de este concepto de traducción, el segundo elemento central de mi trabajo de reconceptualización se refiere específicamente a la noción de espacio. Lefebvre nos invita a considerar el espacio o, más bien dicho, los espacios específicos, a través de su diversidad y su multiplicidad totales. Rechazando el espacio abstracto - «formal y cuantificado» que niega las diferencias provenientes de la naturaleza y del tiempo, así como las «de los cuerpos, de las edades, de los sexos y de las etnias» y que además "funciona positivamente en relación con sus implicaciones: las técnicas, las ciencias aplicadas, un saber relacionado a un poder» (Lefebvre, 1974: 62-63)—, Lefebvre propone una teoría unitaria del espacio que pueda contener «el espacio lógico-epistemológico, el espacio de la práctica social, el [espacio] que ocupan los fenómenos sensibles, sin excluir lo imaginario, los proyectos y proyecciones, los símbolos, las utopías» (Lefebvre, 1974: 19). En aras de acercarse a este espacio unitario, el autor francés propone conceptualizar el espacio en tres niveles interdependientes: las representaciones del espacio (asimilación parcial del espacio a un sistema lingüístico elaborado intelectualmente), la práctica espacial y los espacios de representación. El primero, elemento dominante del trinomio, se relaciona con "conocimientos, signos, códigos y relaciones frontales [oposiciones binarias]» y es el espacio «de los eruditos, de los urbanistas y de los tecnócratas» (Lefebvre, 1974: 43 y 48). La práctica espacial comprende «la producción y la reproducción, lugares específicos y conjuntos espaciales propios a cada formación social» (Lefebvre, 1974: 48). En este nivel de aprehensión, la idiosincrasia de un espacio se relaciona íntimamente con sus usuarios habitantes. Finalmente, Lefebvre nos propone las representaciones del espacio que corresponden al espacio «vivido a través de las imágenes y símbolos que lo acompañan», representaciones que «cubre el espacio físico utilizando simbólicamente sus objetos» (Lefebvre, 1974: 49). A través de este tríptico conceptual, ninguna de las formas cognitivas queda excluida, ya que los tres niveles propuestos corresponden bastante bien al Erklären (el conocimiento de tipo explicativo), la práctica como modalidad de conocimiento ${ }^{3}$ y el Verstehen o hermenéutica. Lefebvre se interesa explícitamente por las relaciones que entretienen estos niveles diferentes. Así, a través del concepto de código espacial ${ }^{4}$, el autor subraya, además de lo que considera como un dominio actual de lo discursivo sobre la práctica, cómo este código se produce de manera dialógica entre los diferentes niveles del espacio que hemos definido anteriormente.

A partir de esta visión dialógica del espacio a través de sus niveles diferentes, he acuñado la expresión «dispositivo de desarrollo territorial». Basándome en el concepto foucaultiano de dispositivo, parcialmente reformulado por Jäger como «contexto, en constante evolución, de elementos contenidos en el habla y en el pensamiento —en la acción y en la materialización—》 (Jäger, 2001: 93),

3. Véase el segundo apartado.

4. El código espacial es «un lenguaje común en la práctica y en la teoría, a los habitantes, arquitectos y científicos» y permite que un espacio sea «vivido, entendido y producido» (Lefebvre, 1974: 59). 
me refiero a conjuntos de elementos discursivos, saberes, acciones y materialidades que definen un intento de desarrollar o transformar un territorio o espacio. Este salto conceptual, del tríptico espacial al dispositivo de desarrollo territorial, parece necesario para salir de una posible lectura estructuralista (en este caso, marxista) del trinomio espacial de Lefebvre con su insistencia sobre la hegemonía del sistema de producción. En efecto, el concepto de dispositivo desarrollado por Foucault en sus estudios genealógicos, donde acentúa «el juego diacrónico de los discursos, prácticas y dispositivos en luchas históricas de poder y para la verdad» (Keller, 2005: 229), permite evitar que se establezca apriorísticamente un centro o una fuente de poder. Luego, con la apropiación del concepto de dispositivo por parte de Jäger, con sus préstamos de la teoría de la actividad (principalmente Vygotsky y Leontjew), «el sujeto que actúa socialmente se convierte en el vínculo entre el discurso y la realidad, un movimiento teórico que modera la severidad del estructuralismo foucaultiano [de los estudios arqueológicos] $]^{5}$ (Meyer, 2001: 43).

Antes de introducir, en el próximo apartado, elementos teóricos que se refieren a los diferentes elementos de los dispositivos de desarrollo territorial, quiero comentar brevemente intentos hechos para informar sobre el desarrollo territorial a partir del marco teórico de Lefebvre. Maharaj y Narsiah se basan implícitamente tanto en el tríptico de Lefebvre, como en el análisis de regímenes de poder-saber de Foucault para construir un marco teórico «realista crítico» capaz de informar sobre la recomposición territorial de África del Sur. Subrayando el valor de la noción de representación del espacio para el estudio de la nueva geografía regional de África del Sur, los autores observan que, en el enfoque de Lefebvre, falta «el reconocimiento del papel de los discursos políticos en el proceso [de producción del espacio]" (Maharaj y Narsiah, 2005: 82). Para paliar el no-reconocimiento del juego de poder implicado en la producción del espacio, los autores se refieren a la noción de conocimiento de Foucault, conocimiento «fundado sobre un discurso responsable del desarrollo y el mantenimiento de un régimen de poder» (Maharaj y Narsiah, 2005: 82). Denis Retaillé, al acuñar el concepto de «espacio móvil», un espacio epistemológico destinado a captar la producción variada y cambiante de «territorios redes», se apoya también en las aportaciones de Lefebvre. Compartiendo con este último la dura crítica del espacio abstracto — «homogéneo pero disociado de los principios societales, o más grave [...], únicamente conforme al principio de mínimo coste económico para un mejor rendimiento en potencia que está culturalmente marcado»—, Retaillé defiende la intrínseca movilidad del espacio, o sea, el hecho que su «referencial material (terrestre) o conceptual (matemático en nuestra tradición) sólo representa el resultado de una fijación por parte de la experiencia interrumpida» (Retaillé, 2005: 189 y 176). Con el espacio móvil, se enfatiza la producción de representaciones del espacio (a través del juego entre los diferentes referenciales) y nos acercamos al espacio de

5. Esta precisión de mi parte proviene de la lectura de la trayectoria de Foucault defendida por Dreyfus y Rabinow (1982) y por Castro (2006). 
las prácticas concretas. Finalmente, los espacios de representación despliegan toda su importancia en el salto programático del espacio móvil —un espacio epistemológico — al espacio legítimo, verdadero proyecto político. Finalmente, quiero subrayar cómo tanto la crítica del espacio abstracto, neutro y transparente, como los diferentes niveles del tríptico espacial de Lefebvre, han sido utilizados por diferentes autoras feministas. Blunt y Rose observan que el espacio transparente, abstracto y presuntamente universal, en tanto que «acceso directo hacia la verdad de los objetos», permite formular alegaciones universalistas o esencialistas sobre la naturaleza de la mujer (Blunt y Rose, 1994: 5). En relación con el efecto liberador de los espacios de representaciones, Robinson estima que éstos sugieren «una espacialidad que se basa en recursos culturales e históricos» y permiten "otras posibilidades de vivir en espacios, diferentes de las dictadas por el orden dominante, o sea por las representaciones del espacio» (Robinson, 2000: 297).

\section{Feminismo, postcolonialismo y teoría no representacional:} de la deconstrucción a la complejidad y a lo político

Después de haber dibujado, en sus grandes rasgos, un marco teórico apto para captar los procesos que tienen lugar entre los diferentes actores y niveles espaciales en los proyectos de cooperación al desarrollo territorial, presento aportaciones principalmente de tres áreas de estudio (los estudios feministas y postcoloniales, así como la perspectiva no representacional) que informan sobre la naturaleza de las representaciones y el valor ontológico y epistemológico de las prácticas.

\section{Las representaciones como "estructuras estructuradas y estructuradoras» ${ }^{6}$}

El lenguaje y sus principales elementos productores de sentido, los discursos y las representaciones, se consideran, por parte de numerosos estudiosos feministas y postcoloniales críticos, entidades dinámicas — relacionadas con un contexto político, cultural y social específico-, que poseen un poder transformativo, tanto sobre la realidad material como sobre la constitución de los sujetos y sus relaciones (Mills, 2005; Lees, 2004). Blunt observa, por ejemplo, que las aportaciones del postestructuralismo sobre la textualidad - principalmente la teoría del discurso inspirada en el trabajo de Foucault - desplazan la "celebración humanista del sujeto individual hacia un compromiso más discursivo y complejo con la subjetividad misma» (Blunt, 1994: 53). Sobre las relaciones entre los sujetos, es bien conocido que Said ha demostrado — de forma quizás esquematizada y esencialista—

6. La "estructura estructurada y estructuradora» es una expresión formulada por Bourdieu para definir el concepto de habitus (Painter, 2000; Keller, 2005). Utilizo dicha expresión para subrayar el carácter construido del lenguaje, así como su efecto performativo sobre la realidad material. 
cómo las pautas de relaciones entre Occidente y Oriente han sido construidas sistemáticamente a través de la producción de un discurso particular: el orientalismo (Said, 1978). Paralelamente, desde la perspectiva feminista, se ha demostrado la construcción discursiva de la masculinidad y la feminidad (Rose, 1993). Finalmente, en relación con la coconstitución discursiva de la realidad material, Reiss nos invita a no "considerar los discursos como grupos de signos, sino como prácticas que sistemáticamente forman el objeto del cual hablan» (Reiss, 1982: 29). Yapa propone la noción de "materialismo discursivo no esencialista» (Yapa, 1997: 718) y Fairclough observa que se debe considerar «la semiosis como un elemento irreducible de todo proceso material social» (Fairclough, 2003: 205).

Estas diversas observaciones sobre la naturaleza y los efectos de lo discursivo me parecen abogar por una sensibilidad hacia una serie de nociones centrales en la geografía postmoderna de carácter crítico: el sujeto descentrado y la interseccionalidad, el papel ideológico de los conceptos y, especialmente, de los conceptos dicotómicos y la performatividad de los discursos. El sujeto descentrado y la interseccionalidad nos invitan a contemplar, en nuestras investigaciones, la inestabilidad del (auto)conocimiento y la (auto)definición llamados «identidad», así como la pluralidad de posiciones discursivas que nos permitan enunciarlo. Rich subraya que el «siempre» (la esencialización) encubre lo que realmente es importante: «el cuándo, dónde y bajo qué condición un enunciado ha sido verdadero" (Rich, 2003 [1983]: 31). Por lo que se refiere a la construcción de conceptos y, especialmente, al pensamiento dualístico presente en dicha actividad, reenvío el lector al estudio de Gilliane Rose (1993) en relación con la influencia continua de la ideología de género, de clase y de raza en el establecimiento de las principales categorías utilizadas en geografía (sujeto/objeto, espacio público/espacio privado, producción/reproducción, el lugar, el espacio-tiempo, etc.). Aquí presento dos propuestas teóricas para salir de una forma de conceptualización esencialista y excluyente que tiene una cierta correspondencia con lo que Retaillé llama «la regla de exclusividad» ${ }^{7}$, que rige la concepción abstracta y dominante del espacio en la geografía (Retaillé, 2005: 187). La primera se refiere al enfoque de inspiración gramsciana adoptado por Said en Culture and Imperialism (1993), donde, según Gregory, el autor palestino adopta "una lectura contrapuntual» que deja espacio para la conexión y la yuxtaposición espacial, así como para una mayor diversidad de relaciones de poder, más allá de la mera opresión y sumisión que caracterizaba Orientalism (Gregory, 2000: 325). Con este enfoque, las oposiciones fundamentadas territorialmente (las geografías excluyentes del Nosotros/Vosotros) se hacen menos estrictas y el espacio se considera como plural. La segunda propuesta es la del «espacio paradójico» propuesto por De Lauretis como la imaginación espacial apta para promover el combate feminista (Rose, 1993). Éste es un espacio multidimensional, cambiante y contingente, es paradójico porque

7. «Dans l'espace abstrait dominant règne une règle consensuelle, celle de l'exclusivité qui fait qu'il ne peut y avoir deux objets en un même lieux» (Retaillé, 2005: 187). 
acepta la simultaneidad de espacios que, representados en dos dimensiones, serían considerados excluyentes. Las espacialidades complejas - fuera de la lógica euclidiana- que acoge este espacio nos hablan del poder y de la resistencia, así como del reconocimiento de la diferencia (Rose, 1993). Estas dos propuestas que relacionan el espacio conceptual (el estar dentro o fuera de una cierta categoría) y la concepción del espacio perceptible tienen una cierta correspondencia con el «espacio móvil» de Retaillé, así como con el «espacio diferencial $\aleph^{8}$ de Lefebvre. Finalmente, en relación con la performatividad de lo discursivo, defiendo que éste no se puede considerar como separado de lo material y que esta relación pasa inexorablemente a través de prácticas, tema que discuto a continuación.

\section{El sentido práctico y su relación con las representaciones}

$\mathrm{Si}$, por una parte, estimo necesario averiguar el papel de las prácticas en el continuo que forman los discursos, las prácticas y la materialidad (o mundo objetual), quiero también insistir sobre las especificidades de este nivel ontológico, aspecto sobre el cual se ha centrado la perspectiva no representacional $^{9}$. De forma más prosaica, puesto que mi objetivo es el de proponer una reconceptualización de la cooperación al desarrollo territorial, así como un marco metodológico para acercarse a ésta, resulta crucial abordar las acciones y las prácticas entendiendo que la cooperación es una intervención, un acto voluntarista.

A partir de una crítica del "giro lingüístico» en la geografía humana que excesivamente se centraba en las representaciones (Latham, 2003) — consideradas como formas estables y no como el resultado de un esfuerzo «situado en un proceso contextual específico de negociación social» (Curt citado por Thrift, 1996: 8) —, diversos autores, en su mayoría anglosajones, han prestado una atención particular a las prácticas. Éstas permiten contemplar los estados «prelingüísticos» $\mathrm{y}$ «encarnados» ${ }^{10}$ que dan una cierta inteligibilidad a la acción humana (Thrift, 1996: 9). Con la perspectiva no representacional, se intenta reconocer el papel del flujo de la vida cotidiana al mismo tiempo que se evita la purificación o sumisión de los diversos órdenes prácticos por/a teorías representacionales (de tipo sociológico, psicológico u otras). Compartiendo con los postestructuralistas la visión del sujeto descentrado, inestable y procesual,

8. La noción de "espacio diferencial» representa un espacio que emerge como alternativa y resistencia frente a la hegemonía de un cierto espacio dominante (Lefebvre, 1974).

9. La perspectiva no representacional o Non-Representational Theory es un ensamblaje de una variedad de inciertos teóricos de la teoría postestructuralista (Foucault, Deleuze, Guatarri, etc.), de la Actor-Network Theory, de teorías de la acción (Bourdieu, Merleau-Ponty, etc.) y del interacionismo simbólico (Blumer, Shotter, etc.) (Thrift, 1996; Smith, 2003; Netto, 2008).

10. Reenvío a Rose et al. (2010) para un ejemplo de utilización del enfoque no representacional en aras de reconocer el papel de los sentimientos y los afectos en la creación del ambiente particular formado por un centro comercial en Milton Keynes. 
este enfoque considera las representaciones como «islas en el mar de nuestra comprensión práctica y no formulada del mundo» (Taylor citado por Thrift, 1996: 10). En él, se aboga por la naturaleza performativa del mundo humano y se insiste sobre la inteligibilidad práctica y la comprensión inarticulada (sin referencia estática) «precisamente porque éstas forman los antecedentes/ prerrequisitos a través de los cuales las representaciones se vuelven comprensibles» (Smith, 2003: 68). La perspectiva no representacional, en definitiva, busca «nuevas vías de conocer que puedan abrir nuevas formas de políticas y éticas liberadoras» (Jones, 2008: 1603). Además de ser una propuesta ontológica que devalúa el papel de las representaciones y que sitúa las prácticas $-\mathrm{y}$ entonces los cuerpos, los sentimientos y las interacciones humanas- en el centro de sus intereses, el enfoque no representacional apuesta por una cierta experimentalidad metodológica apta para captar la naturaleza cambiante, contextual y siempre negociada del mundo que dibuja a nivel teórico. Así, el concepto de performance puede, según Thrift y Dewsbury, cambiar las prácticas académicas de dos formas: $a$ ) "cambiando lo que consideramos como método», "ofreciendo un campo entero de técnicas para hacer del mundo una entidad viva, técnicas que extiendan la gama de las investigaciones actuales y que provean medios para percibir nuevas formas de conocimiento" (Thrift y Dewsbury, 2000: 424); b) «poniendo énfasis en el hecho que la performance es, por sí misma, una forma de conocimiento, una inteligencia en acción» (Thrift y Dewsbury, 2000: 425).

\section{Conclusiones}

En el presente artículo, he intentado dibujar una vía para considerar los proyectos de cooperación al desarrollo territorial bajo el concepto de construcción implicando representaciones, prácticas y valores culturales. A través del concepto de traducción, plural y no apriorísticamente previsible, así como con una atención por las dinámicas y las tensiones que forman el espacio — producto sumamente social—, he intentado abrir el campo del desarrollo territorial a un complejo juego de influencias y de posibilidades. No se trata, pues, de considerar únicamente los procesos participativos en la cooperación como un juego entre los discursos de los actores. En efecto, si nos quedamos a un nivel de "economía de las ideas», numerosos estudiosos postcoloniales nos han advertido que la hegemonía discursiva del paradigma occidental y modernizador difícilmente se podía rebatir. De hecho, Mohanty define la colonización como «un proceso que invariablemente implica una relación de dominación estructural y una supresión discursiva o política de la heterogeneidad de lo(s) sujeto(s) en cuestión» (Mohanty, 2003 [1988]: 50-51). Una vía para evitar esta colonización ontológica y epistemológica del «Otro» es la de reconocer el papel de las prácticas en su total diversidad y obertura a los juegos creativos. Otro tipo de colonización que quiero superar es la del ser sobre los devenires. Latour nos explica que lo social, en su entendimiento, es procesual y, por lo tanto, abierto, y que la «realidad social» que la sociología 
tradicional considera como un objeto estable de estudio sólo representa redes temporalmente cristalizadas por el poder (Latour, 2005).

En definitiva, lo que propongo de forma todavía fragmentaria es la consideración de la «antropo-lógica del espacio» (Peemans y De Rijck, 2004: 7) en los proyectos de cooperación al desarrollo. Ésta sólo se puede desvelar parcialmente considerando las representaciones como procesos complejos de creación de sentido, las prácticas como formas válidas de conocimiento - donde el cuerpo introduce diferencias-y los valores como algo no esencialista y, por lo tanto, como producto de una negociación.

\section{Referencias bibliográficas}

BLunT, Alison (1994). «Mapping authorship and authority: Reading Mary Kingsley’s Landscape Descriptions». En: Blunt, Alison y Rose, Gillian (eds.). Writing women and space: Colonial and Postcolonial Geographies. Nueva York y Londres: The Guilford Press, 51-72.

Buunt, Alison y Rose, Gillian (1994). «Introduction: Women's Colonial and postcolonial Geographies». En: Blunt, Alison y Rose, Gillian (eds.). Writing women and space: Colonial and Postcolonial Geographies. Nueva York y Londres: The Guilford Press, 1-25.

Calderón VÁzquez, F.J. (2008). Guía de orientaciones para la evaluación y seguimiento de proyectos de desarrollo. Edición electrónica gratuita. Texto completo en www.eumed.net/libros/2008b/411/.

CAstro, Edgardo (2006). «Michel Foucault: sujeto e historia». Tópicos, 14, 171-183. [http://www.scielo.org.ar/scielo.php?script=sci_arttext\&pid=S1666-48X200600 0100008\&lng=es\&nrm=iso; consulta: 25 de abril 2010]

Deleuze, Gilles y Guattari, Félix (1972). L'Anti-Oedipe: capitalisme et schizophrénie. París: Les Éditions de Minuit.

Dreyfus, Hubert L. y Rabinow, Paul (1982). Michel Foucault: Beyond structuralism and Hermeneutics. Brighton: The Hervester Press Limited.

FairClough, Norman (2003). Analysing Discourse. Textual analysis for social researcher. Londres y Nueva York: Routledge.

Fish, Stanley (1980). Is there a text in this class?: The authority of interpretative communities. Cambridge y Londres: Harvard University Press.

Garfinkel, Harold (1967). Studies in Ethnometodology. Cambridge: Polity Press.

Gómez Galán, M. y Sáinz Ollero, H. (2003). El ciclo del proyecto de cooperación al desarrollo. La aplicación del marco lógico. Madrid: CYAN, SA.

Gregory, Derek (2000). «Edward Said's imaginative geographies». En: Crang, Mike y Thrift, Nigel (eds.). Thinking Space. Londres: Routledge, 302-348.

JäGER, Siegfried (2001). «Discurso y conocimiento: aspectos teóricos y metodológicos de la crítica del discurso y del análisis de dispositivos». En: WodaK, Ruth y Meyer, Michael (comps.). Métodos de análisis crítico del discurso. Barcelona: Gedisa, 61-100.

JoNEs, Owen (2008) «Stepping from the wreckage: Geography, pragmatism and antirepresentational theory». Geoforum, 39, 1600-1612.

Keller, Reiner (2005). «Analysing Discourse. An aproach From sociology of Knowledge». Forum Qualitative Socialforshung, 6 (3). [http://www.qualitativeresearch.net/fqs-texte/3-05/05-3-32-e.htm; consulta: 14 de marzo de 2010] 
Latham, Alan (2003). «Research, performance, and doing human geography: some reflections on the diary-photograph, diary interview method». Environment and Planning A, 35, 1993-2017.

Latour, Bruno (1996). Aramis or the love of technology. Cambridge y Londres: Harvard University Press.

- (2005). Reassembling the Social. An introduction to Actor-Network-Theory. Nueva York: Oxford University Press Inc.

Lefebvre, Henri (1974). La production de l'espace. París: Anthropos.

LEEs, Loretta (2004). "Urban geography: discourse analysis and urban research». Progress in Human Geography, 28 (1), 101-107.

Maharaj, Brij y Narsiah, Sagie (2005). «La nouvelle géographie régionale de l'Afrique du Sud post-apartheid». En: Antheaume, Benoît y Giraut, Frédérique (eds.). Le territoire est mort. Vive les territoires! Une (re)fabrication au nom du développement. París: IRD Éditions, 79-107.

Massey, Doreen (2003) «Imagining the field». En: Pryke, M.; Rose, G. y WhatMORE, S. (eds.). Using Social Theory. Thinking through Research. Londres: Sage Publications Ltd., 71-88.

Mawdsley, Emma; Townsend, Janet; Porter, Gina y Oakley, Peter (2002). Knowledge, power and development agendas: NGOs North and South. Oxford: INTRAC.

Meyer, Michael (2001). «Entre la teoría, el método y la política: la ubicación de los enfoques relacionados con el ACD». En: Wodak, Ruth y Meyer, Michael (comps.). Métodos de análisis crítico del discurso. Barcelona: Gedisa, 35-59.

Mills, Sara (2005). Gender and Colonial Space. Manchester y Nueva York: Manchester University Press.

Mohanty, Chandra Talpade (2003 [1988]). «Under Western Eyes: Feminist Scholarship and Colonial Discourses». En: Lewis, Reina y Mills, Sara (eds.). Feminsist Postcolonial Theory: A Reader. Edimburgo: Edinburgh University Press Ltd., 49-74.

Netтo, Vinicius (2008). "Practice, space and the duality of meaning». Environment and Planning D, 26 (2), 359-379.

Painter, Joe (2000). «Pierre Bourdieu». En: Crang, Mike y Thrift, Nigel (eds.). Thinking Space. Londres: Routledge, 239-259.

Peemans, J-P. y De Rijck, K. (2004). Mobilité et paysage: les rapports entre espaces urbains, périurbains et ruraux: convergences et divergences des regards du développement et de l'urbanisme. Presses Universitaires de Louvain.

ReIss, Timothy J. (1982). The discourse of modernity. Ithaca: Cornell University Press.

Retaillé, Denis (2005). «L'espace mobile». En: Antheaume, Benoît y Giraut, Frédérique (eds.). Le territoire est mort. Vive les territoires! Une (re)fabrication au nom du développement. París: IRD Éditions, 175-201.

- (2007) «Quel est l'impacte de la mondialisation sur le développement local?». Les Cahiers d'Outre-Mer, 238, 167-183.

Rich, Adrienne (2003 [1983]). «Notes towards a Politics of Location». En: Lewis, Reina y Mills, Sara (eds.). Feminsist Postcolonial Theory: A Reade. Edimburgo: Edinburgh University Press Ltd., 29-42.

Robinson, Jenny (2000). «Feminism and the spaces of transformation». Transactions of the Institute of British Geographers, 25 (3), 285-301.

Rose, Gillian (1993). Feminism and Geography: The Limits of Geographical Knowledge. Mineapolis: University of Mineapolis Press. 
Rose, Gillian; Degen, Monica y Basdas, Begum (2010). «More on "big things": building events and feelings». Transactions of the Institute of British Geographers, 35, 334-349.

SAID, Edward W. (1978). Orientalism. New York: Pantheon Books.

- (1983). The world, the text, and the critic. Londres: Harvard University Press.

- (1993). Culture and Imperialism. Nueva York: Knopf.

SMITH, Richard G. (2003). «Baudrillard's nonrepresentational theory: burn the signs and journey without maps». Environment and Planning D, 21 (1), 64-84.

Thrift, Nigel (1996). Spatial Formations. Londres: Sage.

Thrift, Nigel y Dewsbury, John-David (2000). «Dead geographies — and how to make them live». Environnement and Planning D, 18 (4), 411-432.

YAPA, Lakshman (1997). «Reply: Why Discourse Matter, Materiality». Annals of Association of American Geographers, 87 (4), 717-722. 